\title{
Silicon Photonic Cross-bar Switching and Wavelength Conversion in a WDM Network Testbed (Project Report CIAN2-3-Y1)
}

\section{SHAYAN MOOKHERJEA}

Department of Electrical and Computer Engineering, University of California, San Diego, MC 0407 La Jolla CA 92093-0407 USA

Email:smookher@ucsd.edu

\section{Introduction and Goals}

A partnership between UCSD, Cornell, UC Berkeley, Caltech and the University of Arizona collaborated with Sandia in a silicon photonics multiproject wafer (MPW) project in 2013. This was a project "Silicon Manufacturing" under the Thrust 2 "Subsystem Integration and Silicon Nanophotonics" of the NSF-funded Center for Integrated Access Networks. This was the first group of external university users of Sandia's silicon photonics foundry fabrication capabilities. The goal of this research was to design, fabricate and test chip-scale opto-electronic network systems for access and data networks. This multi-year project supports the overall vision of the Center that innovative optical components will need to be designed, integrated and co-manufactured for serving the growing bandwidth needs of optical access and data networks in an energy-efficient and scalable way.

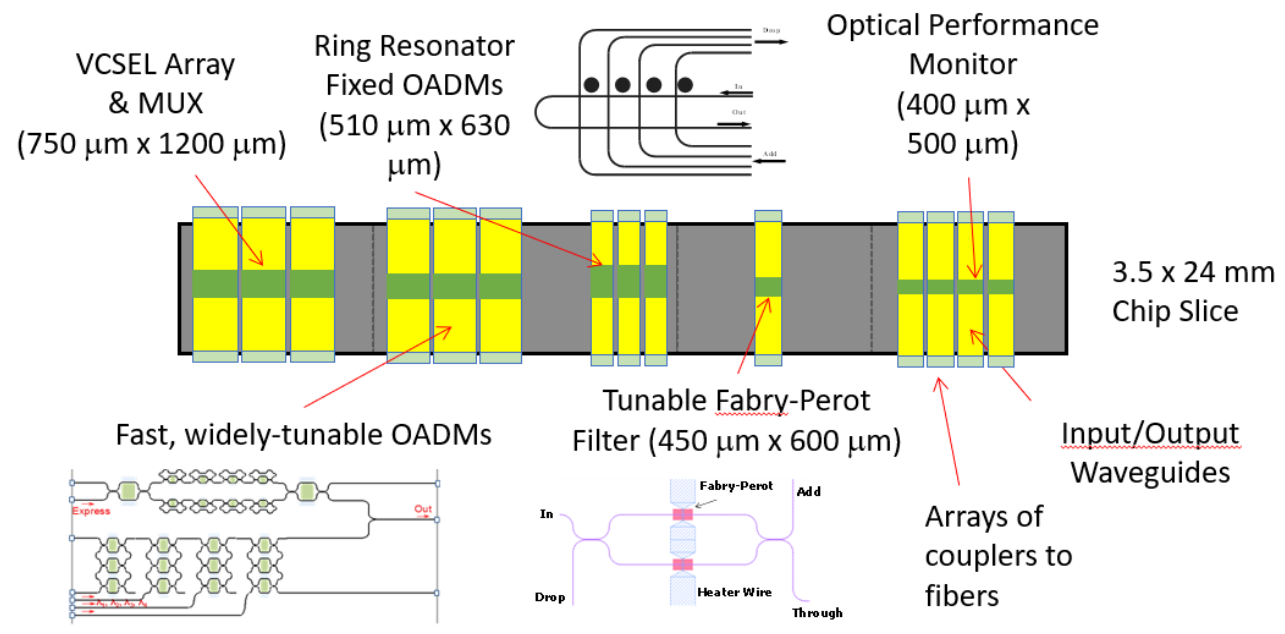

Figure 1 Initial floor-planning of a multi-project wafer (MPW) fabrication run to study integrated photonics devices for optical access and data networks.

Figure 1 shows the floor-planning of the initial MPW chip, which included a variety of optical structures that can be useful for optical communications. Figure 2 shows the process flow for participating in the MPW, including establishing the paperwork with Sandia and obtaining a design manual prepared 
by Sandia scientists, preparing CAD designs for fabrication, and submitting the GDS for fabrication. Fabricated microchips were returned to the designers in several rounds of deliverables after a period of a few months up to more than a year after design submission, depending on the complexity of the designs and processing and other higher-priority projects underway at the foundry. Passive chips (silicon only, without dopants, germanium, or metals) were generally received earlier, typically within six months.

(a)

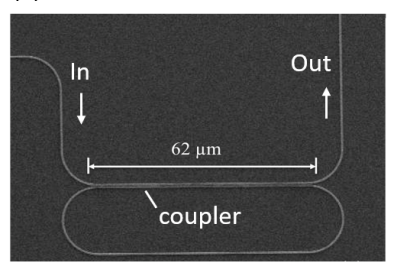

(b)

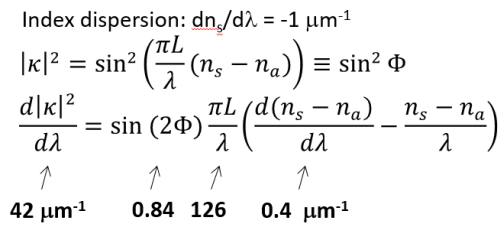

(c)

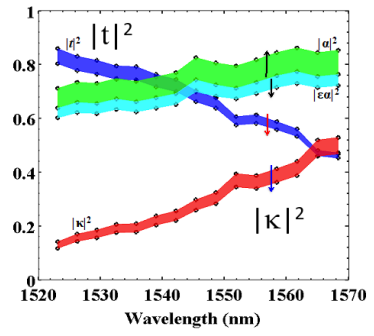

Figure 2 Studying the dispersion of a directional coupler using a microring resonator. The dispersion of the coupler can be many times higher than the dispersion of the refractive index, and leads to a significant variation in the coupling coefficient versus wavelength.

\section{Activities}

Some of the structures in the MPW design used single or coupled silicon microrings. We experimentally studied the dispersion of directional couplers using a microring coupled to a waveguide, as shown in Figure 2 [1]. An accurate knowledge of dispersion is also necessary for a wide range of integrated photonics devices, such as coupled-microring filters [2], some of which are included in the layout shown in Figure 1.

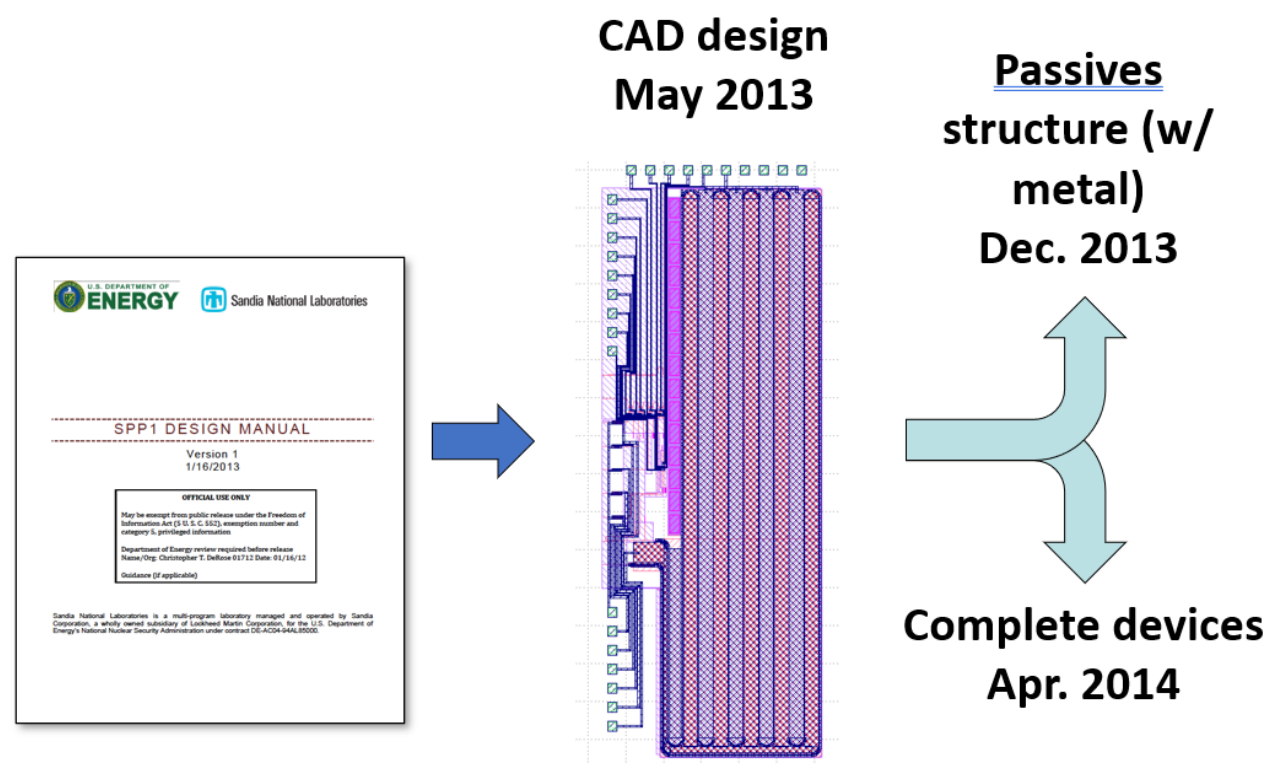

Figure 3 Process flow for participation in the MPW. 
The design flow for the MPW fabrication is schematically shown in Figure 3. While waiting for the MPW chips to be delivered, we also tested some silicon photonics chips that were previously designed and fabricated by Sandia with the wavelength division multiplexed (WDM) optical network at UC San Diego [3]. A typical silicon photonic $2 \times 2$ waveguide switch, based on a MachZehnder interferometer with thermo-optic heaters, can effectively switch data signals from a "cross" port to a "bar" port but requires a precisely-controlled analog voltage. As shown in Fig. 4(b), a voltage of $4.24 \mathrm{~V}$ was required to switch the signal from the cross to the bar port. It is usually more convenient to generate a constant voltage from a digital output pin of an electronic driver and use pulse-width modulation to achieve the same switching effect, as shown in Fig. 4(c). An inexpensive electronics driver circuit or digital-to-analog converter (DAQ) module has many more digital ports than analog output ports, and thus, can potentially control many more heaters in a multi-component photonic circuit if driven from the digital ports.

(a)

$$
\begin{aligned}
& \text { Cross port } \\
& \text { No voltage }
\end{aligned}
$$

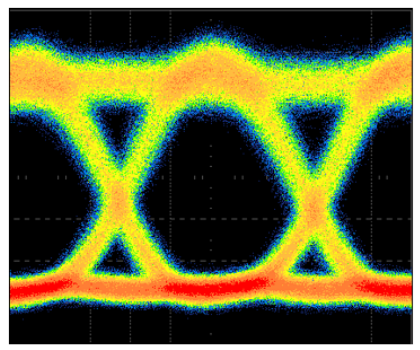

10 Gigabit/s eye

ITU-T Channel 24 in MORDIA (b)

Bar port

$4.24 \mathrm{~V}(\mathrm{DC})$

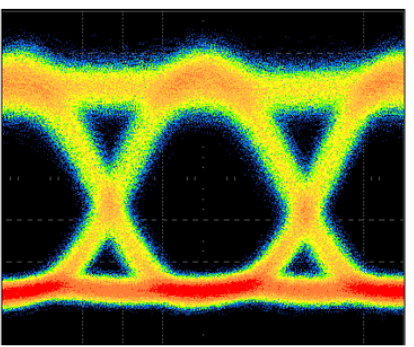

Needs an analog

output pin. (c) Bar port $15 \mathrm{MHz}, 10 \mathrm{~V}, 42 \% \mathrm{dc}$

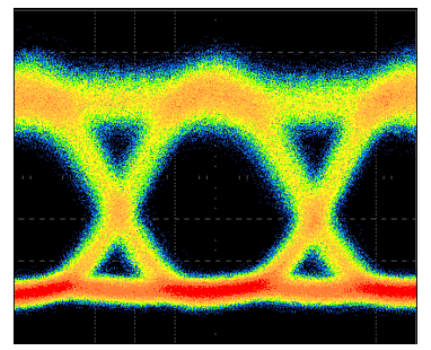

Needs a digital output pin.

Figure 4 (a) Eye diagram of a $10 \mathrm{~Gb} / \mathrm{s} \mathrm{NRZ} \mathrm{data} \mathrm{in} \mathrm{the} \mathrm{WDM} \mathrm{MORDIA} \mathrm{optical} \mathrm{network} \mathrm{testbed}$ passed through the cross-port of a $2 \times 2$ thermo-optic silicon photonic switch. (b) Eye diagram of the switched signal appearing in the bar port, with the application of a 4.24V DC signal. (c) Switching was achieved using a pulse-width modulated waveform with $10 \mathrm{~V}$ peak voltage.

This thermo-optic cross-bar switch designed by Sandia researchers used an innovative design, in which the heaters consisted of doped segments of bent silicon waveguides. Light propagates along the outer edge of a waveguide bend with a small radius of curvature, allowing dopants to be placed on the inner edge without incurring excessive optical loss. These dopants can be connected to wires, and function as a micro-heater placed in close proximity to the optical mode. Therefore, the thermo-optic switch can be fast and efficient. A digital driver is needed to best utilize the fast switching capability of these heaters. 


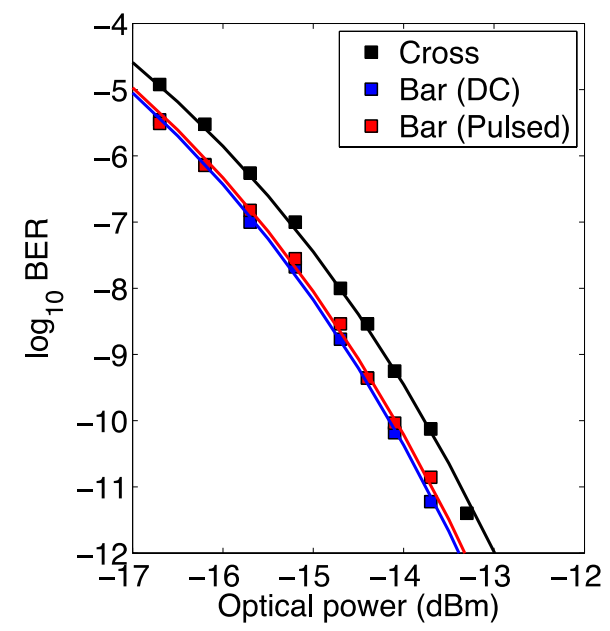

Figure 5 Bit error rate (BER) versus optical power for a Mach-Zehnder Interferometer (MZI) silicon photonic $2 \times 2$ switch.
Figure 5 shows the bit-error-rate (BER) curves versus optical power for the three cases shown in Figure 4. The cross port as measured at $0 \mathrm{~V}$ (bias-free configuration) has a minor penalty because without applying any voltage, the switch was not perfectly aligned to the optimal cross configuration of the Mach-Zehnder interferometer (MZI). This is typical in silicon photonics, because of fabrication imperfections which can cause a path-length imbalance in optically long structures such as MZI's. This test structure included heaters on only one arm of the MZI, and therefore, it was not possible to correct for the small error (by heating

the other arm). Both the bar configurations achieved the same performance, showing that the digital output of a controller could be used for switching.

\section{Progress and Impact}

A four-wave mixing (FWM) experiment was performed to show that silicon photonic microchips can be used in wavelength conversion of modulated data in a WDM network. Two channels from the MORDIA WDM network testbed, each modulated with $10 \mathrm{~Gb} / \mathrm{s} \mathrm{NRZ}$ data, were converted using a continuouswave pump to different channels, as shown in Figure 6. The input channels were aligned to different passbands of the $\mathrm{CROW}$, and the $\mathrm{CW}$ pump was on a different passband. Idlers were generated based on the energy conservation relationships $\omega_{\mathrm{I}}{ }^{(\mathrm{n})}=2 \omega_{\mathrm{P}}-\omega_{\mathrm{S}}{ }^{(\mathrm{n})}$ where $\mathrm{n}=1,2, \ldots$ enumerates the number of input signals (two in this example). The idlers were also resonant with the CROW. FWM with a CW pump is difficult to achieve in a conventional silicon waveguide, because the required optical powers to achieve converted power levels in the milliwatt (or at least hundreds of microwatt) regime are very high (typically, greater than $0.5 \mathrm{~W}$ ), and incurs substantial two-photon absorption loss. Some method of increasing the effective nonlinearity is therefore required. Here, the device used to perform four-wave mixing was a coupled-resonator optical waveguide (CROW) which was resonant with the pump and each of the signal inputs and idler outputs. Thus, when the experiment in Figure 6 was being performed, a five-fold resonance was achieved and maintained, which is very difficult to do using bulk nonlinear optical crystals. Such a device achieves a very high FWM nonlinearity (nearly 50 times higher than that of a silicon waveguide). 
When the optical power is increased (typically beyond 10-100 $\mathrm{mW}$ ), silicon microring resonators with a modest $\mathrm{Q}$ factor that use directional couplers can exhibit interesting behavior, such as bistability and nonlinear on-off switching [4]. However, these effects are not expected to be significant at milliwatt power levels. Optical amplifiers were used to compensate for the fiber-to-chip coupling loss (about 5-6 dB per facet) but did not impair the performance of the CROW structure itself. Some eye closure is seen in Fig. 6, which is attributed to the relatively high dispersion of the CROW structure.

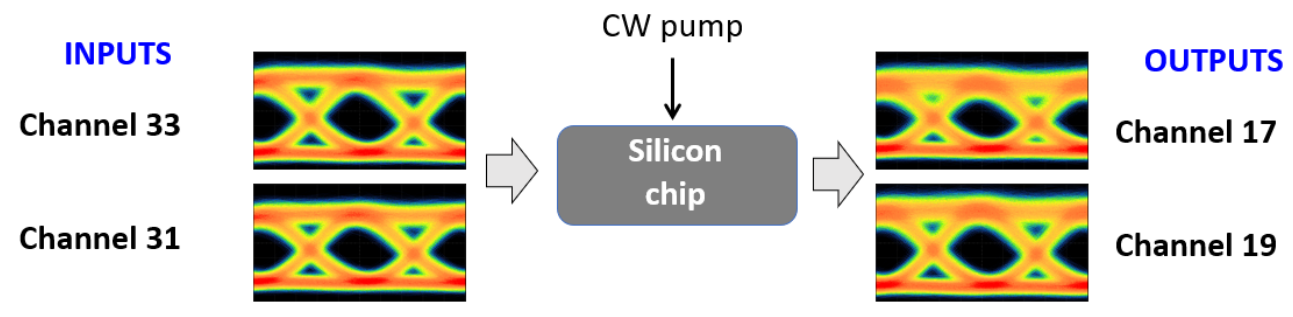

\begin{abstract}
Figure 6 Four-wave mixing (FWM) was used to convert the carrier wavelength of two WDM data channels in the MORDIA testbed to other channels. The FWM was performed using a silicon photonic microchip which contained a coupled-resonator optical waveguide (CROW) structure. Both channels of FWM are phase-matched and all five waves are simultaneously resonantly enhanced in intensity (different passbands of the CROW), which is very difficult to achieve using bulk nonlinear crystals.
\end{abstract}

Previously, we have developed the theory and design principles of coupled microring resonators, also known as Coupled Resonator Optical Waveguides (CROWs). A tight-binding model of band-center propagation in CROWs was formulated [5], [6], and theoretical studies of nonlinear propagation were developed on this basis [7]-[9]. Studies were performed to examine the regions of high dispersion which may be relevant to signal distortion when filtering wideband signals [10], [11] or all-optically tunable dispersion compensation devices [12]

To help in designing structures in silicon photonics, we have developed an extension of coupled-mode theory which is applicable to directional couplers in high index contrast materials, in coupled-waveguide structures [13] and coupled-resonator structures [14]. A collaboration with IBM's silicon photonics group (Dr. W. M. J. Green) has provided insights into silicon photonics fabrication and also supported technical collaboration on fabricating structures at wafer-scale using silicon photonics processing [15], [16], [11]. Dr. Ivan B. Divliansky (Research Scientist, CREOL, Florida) collaborated with us for the electron-beam lithography of long waveguide structures [17], [18]. CROWs of up to 235 coupled resonators were successfully fabricated and measured, and a good agreement was achieved between experiments and theory [16], [19], [20]. 
Impact on Human Resources: One graduate student in the PI's group worked on this topic as part of their education and training. The Center provided extensive opportunities to interact with researchers and students from other groups and universities as well as with researchers from Sandia. We are also grateful to Dr. Vikas Anant for collaboration on cryogenically-cooled detection apparatus, leading to a jointly-authored publication [21].

\section{Subsequent Extensions}

The CROW structure has a nonlinear dispersion relationship [22], and can have other applications including generation of quantum light [23], and developing amplifiers and lasers with novel properties [24]. For example, the resonatorenhanced nonlinearity benefits the generation of entangled photon-pair and heralded single-photon generation using silicon photonics [25]-[27]. Our initial measurements of spontaneous four-wave mixing (SFWM) were on correlated photon-pair generation and heralded single-photon generation. An experimental measurement of entanglement was performed later, using a twophoton interferometer constructed to verify time-energy entanglement [25].

Electronic controllability is also of interest in the CROW structure [28], although the mechanism was different from thermo-optic heating. Carrier driven effects can achieve sub-nanosecond speeds of varying the optical index and absorption. P-N junctions can be incorporated within silicon microrings by doping sections of the waveguide cross-section [29]. Even faster effects can be achieved using ferro-electric materials which decouple the amplitude and phase effects. For example, optically-transparent thin-film ferro-electric materials bonded to silicon waveguides can be used to achieve phase shifters with more than $100 \mathrm{GHz}$ of bandwidth, as was demonstrated in our later work [30].

Microring resonators are sensitive to disorder. Infrared imaging is a useful experimental tool to study light propagation through the length of these structures [17], [31] and distinguish the effects of distributed nanoscale disorder from isolated fabrication faults and design errors. In some applications, a heater for tuning the resonance wavelength is not desirable. We studied potential methods for precisely tuning resonators without heaters. Our method is based on field-induced local oxidation of $\mathrm{Si}$ to $\mathrm{SiO}_{2}$ via a chemical reaction near an electrically-biased conducting atomic-force microscope tip [32]. Scanning probe lithography has previously been used to modify the resonance frequency of a GaAs photonic crystal cavity. A single silicon microresonator can be monitored and controlled more easily [33] than a long CROW device.

Both microring resonator-based optical switching and switching based on the Mach-Zehnder interferometer architecture continue to be an active area of research. The double-ring architecture with thermo-optic tuning using heaters built into the rings was used in an $8 \times 8$ switch device with microsecond scale 
reonfigurability [34]. A combination of rings and Mach-Zehnders with both electro-optic switching and thermo-optic tuning of the bias point was used to demonstrate sub nanosecond $\mathrm{NxN}$ optical switching for $\mathrm{N}=2$ to 16 [35]-[37]. Using current-injection Mach-Zehnder interferometer switching devices in a manufacturing process which includes electronic circuits, IBM has developed $4 \times 4$ and $8 \times 8$ switches integrated with drivers for nano-second reconfigurability[38].

\section{Open-Access Reporting Initiative}

PRAISE: This open-access document is provided in support of our PRAISE (Public Report of Activities, Impact and Subsequent Extensions) initiative. What is it? An open-access document shared with the public which describes the research outcomes of publicly-funded projects. For us, these projects are typically funded by the NSF (National Science Foundation).

\section{References}

[1] R. Aguinaldo, Yiran Shen, and S. Mookherjea, "Large Dispersion of Silicon Directional Couplers Obtained via Wideband Microring Parametric Characterization," IEEE Photon. Technol. Lett., vol. 24, no. 14, pp. 1242-1244, Jul. 2012, doi: 10.1109/LPT.2012.2198639.

[2] J. R. Ong, R. Kumar, and S. Mookherjea, "Silicon microring-based wavelength converter with integrated pump and signal suppression," Opt. Lett., vol. 39, no. 15, p. 4439, Aug. 2014, doi: 10.1364/OL.39.004439.

[3] R. Aguinaldo et al., "Wideband silicon-photonic thermo-optic switch in a wavelength-division multiplexed ring network," Opt. Express, vol. 22, no. 7, p. 8205, Apr. 2014, doi: 10.1364/OE.22.008205.

[4] S. Mookherjea and M. A. Schneider, "The nonlinear microring add-drop filter," Opt. Express, vol. 16, no. 19, p. 15130, Sep. 2008, doi: 10.1364/OE.16.015130.

[5] S. Mookherjea and A. Yariv, "Optical pulse propagation in the tightbinding approximation," Opt. Express, vol. 9, no. 2, p. 91, Jul. 2001, doi: 10.1364/OE.9.000091.

[6] S. Mookherjea and A. Yariv, "Pulse propagation in a coupled resonator optical waveguide to all orders of dispersion," Phys. Rev. E, vol. 65, no. 5, p. 056601, Apr. 2002, doi: 10.1103/PhysRevE.65.056601.

[7] S. Mookherjea and A. Yariv, "Kerr-stabilized super-resonant modes in coupled-resonator optical waveguides," Phys. Rev. E, vol. 66, no. 4, p. 046610, Oct. 2002, doi: 10.1103/PhysRevE.66.046610. 
[8] S. Mookherjea and A. Yariv, "Optical pulse propagation and holographic storage in a coupled-resonator optical waveguide," Phys. Rev. E, vol. 64, no. 6, p. 066602, Nov. 2001, doi: 10.1103/PhysRevE.64.066602.

[9] S. Mookherjea and A. Yariv, "Second-harmonic generation with pulses in a coupled-resonator optical waveguide," Phys. Rev. E, vol. 65, no. 2, p. 026607, Jan. 2002, doi: 10.1103/PhysRevE.65.026607.

[10] S. Mookherjea, D. S. Cohen, and A. Yariv, "Nonlinear dispersion in a coupled-resonator optical waveguide," Opt. Lett., vol. 27, no. 11, p. 933, Jun. 2002, doi: 10.1364/OL.27.000933.

[11] M. L. Cooper et al., "Waveguide dispersion effects in silicon-oninsulator coupled-resonator optical waveguides," Opt. Lett., vol. 35, no. 18, p. 3030, Sep. 2010, doi: 10.1364/OL.35.003030.

[12] S. Mookherjea, "Using gain to tune the dispersion relation of coupledresonator optical waveguides," IEEE Photon. Technol. Lett., vol. 18, no. 5, pp. 715-717, Mar. 2006, doi: 10.1109/LPT.2006.871144.

[13] M. L. Cooper and S. Mookherjea, "Numerically-assisted coupledmode theory for silicon waveguide couplers and arrayed waveguides," Opt. Express, vol. 17, no. 3, p. 1583, Feb. 2009, doi: 10.1364/OE.17.001583.

[14] S. Mookherjea, "Spectral characteristics of coupled resonators," $J$. Opt. Soc. Am. B, vol. 23, no. 6, p. 1137, Jun. 2006, doi: 10.1364/JOSAB.23.001137.

[15] J. R. Ong et al., "Low-power continuous-wave four-wave mixing in silicon coupled-resonator optical waveguides," Opt. Lett., vol. 36, no. 15, pp. 2964-2966, 2011.

[16] M. L. Cooper et al., "235-ring Coupled-Resonator Optical Waveguides," in Conference on Lasers and Electro-Optics 2010, San Jose, California, 2010, p. CTuHH3. doi: 10.1364/CLEO.2010.CTuHH3.

[17] M. L. Cooper, G. Gupta, J. S. Park, M. A. Schneider, I. B. Divliansky, and S. Mookherjea, "Quantitative infrared imaging of silicon-on-insulator microring resonators," Opt. Lett., vol. 35, no. 5, p. 784, Mar. 2010, doi: 10.1364/OL.35.000784.

[18] Y. Shen, I. B. Divliansky, D. N. Basov, and S. Mookherjea, "Electricfield-driven nano-oxidation trimming of silicon microrings and interferometers," Opt. Lett., vol. 36, no. 14, p. 2668, Jul. 2011, doi: 10.1364/OL.36.002668.

[19] S. Mookherjea and M. A. Schneider, "Avoiding bandwidth collapse in long chains of coupled optical microresonators," Opt. Lett., vol. 36, no. 23, p. 4557, Dec. 2011, doi: 10.1364/OL.36.004557.

[20] M. L. Cooper and S. Mookherjea, "Modeling of Multiband Transmission in Long Silicon Coupled-Resonator Optical Waveguides," IEEE Photon. Technol. Lett., vol. 23, no. 13, pp. 872-874, Jul. 2011, doi: 10.1109/LPT.2011.2141657. 
[21] C. Ma, X. Wang, V. Anant, A. D. Beyer, M. D. Shaw, and S. Mookherjea, "Silicon photonic entangled photon-pair and heralded single photon generation with CAR $>12,000$ and $\mathrm{g}^{\wedge}(2)(0)<0006$, , Opt. Express, vol. 25, no. 26, p. 32995, Dec. 2017, doi:

10.1364/OE.25.032995.

[22] S. Mookherjea, "Dispersion characteristics of coupled-resonator optical waveguides," Opt. Lett., vol. 30, no. 18, p. 2406, Sep. 2005, doi: 10.1364/OL.30.002406.

[23] J. R. Ong and S. Mookherjea, "Quantum light generation on a silicon chip using waveguides and resonators," Opt. Express, vol. 21, no. 4, p. 5171, Feb. 2013, doi: 10.1364/OE.21.005171.

[24] S. Mookherjea, "Semiconductor coupled-resonator optical waveguide laser,” Appl. Phys. Lett., vol. 84, no. 17, pp. 3265-3267, Apr. 2004, doi: 10.1063/1.1719278.

[25] R. Kumar, M. Savanier, J. R. Ong, and S. Mookherjea, "Entanglement measurement of a coupled silicon microring photon pair source," Opt. Express, vol. 23, no. 15, p. 19318, Jul. 2015, doi: 10.1364/OE.23.019318.

[26] R. Kumar, J. R. Ong, J. Recchio, K. Srinivasan, and S. Mookherjea, "Spectrally multiplexed and tunable-wavelength photon pairs at $155 \mu \mathrm{m}$ from a silicon coupled-resonator optical waveguide," Opt. Lett., vol. 38, no. 16, p. 2969, Aug. 2013, doi: 10.1364/OL.38.002969.

[27] R. Kumar, J. R. Ong, M. Savanier, and S. Mookherjea, "Controlling the spectrum of photons generated on a silicon nanophotonic chip," Nat Commun, vol. 5, no. 1, p. 5489, Dec. 2014, doi: 10.1038/ncomms6489.

[28] S. Mookherjea, J. R. Ong, X. Luo, and L. Guo-Qiang, "Electronic control of optical Anderson localization modes," Nature Nanotech, vol. 9, no. 5, pp. 365-371, May 2014, doi: 10.1038/nnano.2014.53.

[29] J. R. Ong, R. Kumar, R. Aguinaldo, and S. Mookherjea, "Efficient CW Four-Wave Mixing in Silicon-on-Insulator Micro-Rings With Active Carrier Removal," IEEE Photon. Technol. Lett., vol. 25, no. 17, pp. 1699 1702, Sep. 2013, doi: 10.1109/LPT.2013.2272521.

[30] X. Wang, P. O. Weigel, J. Zhao, M. Ruesing, and S. Mookherjea, "Achieving beyond-100-GHz large-signal modulation bandwidth in hybrid silicon photonics Mach Zehnder modulators using thin film lithium niobate," APL Photonics, vol. 4, no. 9, p. 096101, Sep. 2019, doi: 10.1063/1.5115243.

[31] S. Mookherjea and H. R. Grant, "High dynamic range microscope infrared imaging of silicon nanophotonic devices," Opt. Lett., vol. 37, no. 22, p. 4705, Nov. 2012, doi: 10.1364/OL.37.004705.

[32] Y. Shen, I. B. Divliansky, D. N. Basov, and S. Mookherjea, "Perfect set-and-forget alignment of silicon photonic resonators and interferometers," in Optical Fiber Communication Conference/National 
Fiber Optic Engineers Conference 2011, Los Angeles, California, 2011, p. PDPC3. doi: 10.1364/OFC.2011.PDPC3.

[33] M. Savanier, R. Kumar, and S. Mookherjea, "Optimizing photon-pair generation electronically using a $p-i-n$ diode incorporated in a silicon microring resonator," Appl. Phys. Lett., vol. 107, no. 13, p. 131101, Sep. 2015, doi: 10.1063/1.4932047.

[34] Y. Huang et al., "Multi-Stage $8 \times 8$ Silicon Photonic Switch Based on Dual-Microring Switching Elements," J. Lightwave Technol., vol. 38, no. 2, pp. 194-201, Jan. 2020, doi: 10.1109/JLT.2019.2945941.

[35] L. Lu, L. Zhou, X. Li, and J. Chen, "Low-power $2 \times 2$ silicon electrooptic switches based on double-ring assisted Mach-Zehnder interferometers," Opt. Lett., vol. 39, no. 6, p. 1633, Mar. 2014, doi: 10.1364/OL.39.001633.

[36] L. Lu et al., "\$4\times 4\$ Silicon Optical Switches Based on DoubleRing-Assisted Mach-Zehnder Interferometers," IEEE Photon. Technol. Lett., vol. 27, no. 23, pp. 2457-2460, Dec. 2015, doi: 10.1109/LPT.2015.2470133.

[37] L. Zhou, L. Lu, Z. Guo, and J. Chen, "Silicon 16×16 switch matrix based on dual-ring assisted MZI structures with fast and energy efficient switching," in 2017 IEEE Photonics Society Summer Topical Meeting Series (SUM), San Juan, PR, USA, Jul. 2017, pp. 35-36. doi: 10.1109/PHOSST.2017.8012638.

[38] B. G. Lee et al., "Monolithic Silicon Integration of Scaled Photonic Switch Fabrics, CMOS Logic, and Device Driver Circuits," J. Lightwave Technol., vol. 32, no. 4, pp. 743-751, Feb. 2014, doi: 10.1109/JLT.2013.2280400. 